\title{
A New In-situ Broad Ion Beam, With Energy Range 1 - 500 eV
}

\author{
J.J.L. Mulders, P.H.F Trompenaars, E.G.T. Bosch, R.T.J.P. Geurts \\ FEI Company, Achsteweg Noord 5, 5600MD Eindhoven, The Netherlands
}

The interest in low energy ion beams (typically Ar at $50-500 \mathrm{eV}$ ), is increasing and finds applications in surface clean up, such as removal of hydrocarbons and oxide layers and in fields related to reactive ion etching. Also the removal of $\mathrm{Ga}^{+}$ions and the amorphous layer in a TEM lamella prepared with FIB is interesting. Within the environment of an SEM or DualBeam a new ion source has been constructed. The ion source is based on a narrow gas channel, in which the atoms are converted into ions using direct ionization by the primary electron beam of the system. This local ionization is primarily driven by the electron ionization cross-section of the gas involved, as a function of the primary electron beam energy. Opposite the channel is a surface at potential $-\mathrm{V}$ and at gap distance $\mathrm{d}$ (Figure 1). The resulting field ($\mathrm{V} / \mathrm{d}$ ) between this surface and the channel-output, will induce ion acceleration towards the surface: in this way a stationary broad beam of ions with well-defined energy is created. In this set-up the ion energy and the ion current are decoupled parameters and hence can be chosen each within their practical boundaries. In case the gas type is changed, the ionization only scales with the respective cross-section of the applied gas. The behavior of ion source has been simulated with both Opera simulation software and GEANT4 [1], using the actual geometrical set up and physical data as input. This allows to study the influence of the most relevant parameters, including the geometry and to compare it to measured values -using a method described below- with the aim to optimize the source for its application.

As the low energy ions can induce milling (or sputtering) the local flux can be determined by measuring the amount of material that is sputtered. To this end, a silicon sample has been coated with Pt of high purity using an atomic layer deposition (ALD) process and the thickness is calibrated against an ellipsometer. The normalized EDX net peak intensity ration of PtM and ( $\mathrm{SiK}+\mathrm{PtM})$ are measured and plotted against the real thickness (Figure 2). In this way the local Pt layer thickness is determined with an accuracy of $<1 \mathrm{~nm}$ and lateral resolution $<30 \mathrm{~nm}$.

In experiments with the new source operating with Ar, some of the $23 \mathrm{~nm}$ thick Pt ALD is sputtered typically between 2 and $20 \mathrm{~nm}$. Then, the local net peak intensity ratio is determined at various locations (Figure 3) and translated to the local thickness. This method allows an accurate determination of the ion beam profile, even in the very low energy regime where milling is slow. The profile of the new in-situ ion source (size, shape) has been measured to study the relation between profile, gap distance $\mathrm{d}$ and voltage $-\mathrm{V}$. The result compares favorably with simulations (Figure 4). In addition by integrating the amount of sputtered material over the surface area, the total volume can be calculated and hence the speed of the milling process can be determined. Finally, including the actual ion beam current the data can be compared with, for example, SRIM sputter yields for Ar on Pt.

\section{References:}

[1] Kieft E and Bosch E, J. Phys. D: Appl. Phys. 41 (2008), p. 215310. 


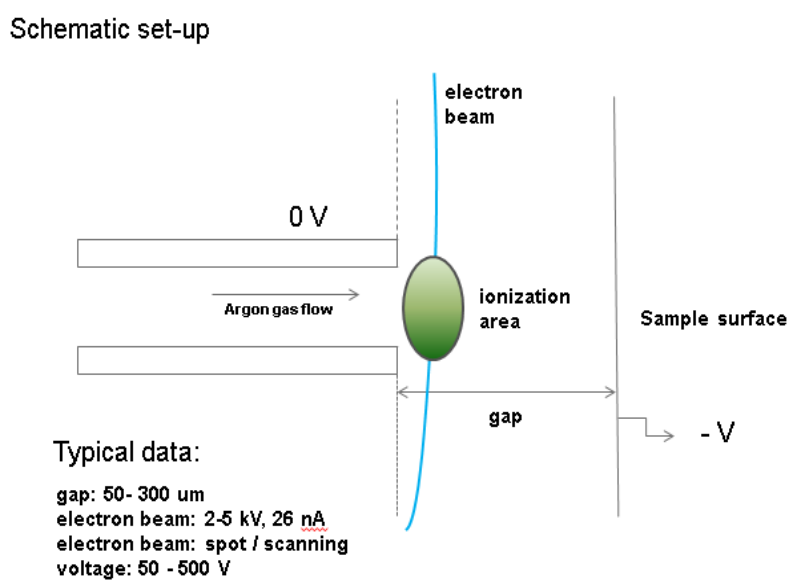

Figure 1: Schematic set up of an in-situ low energy ion source. The field $-\mathrm{V} /$ gap induces acceleration of ions towards the sample surface.

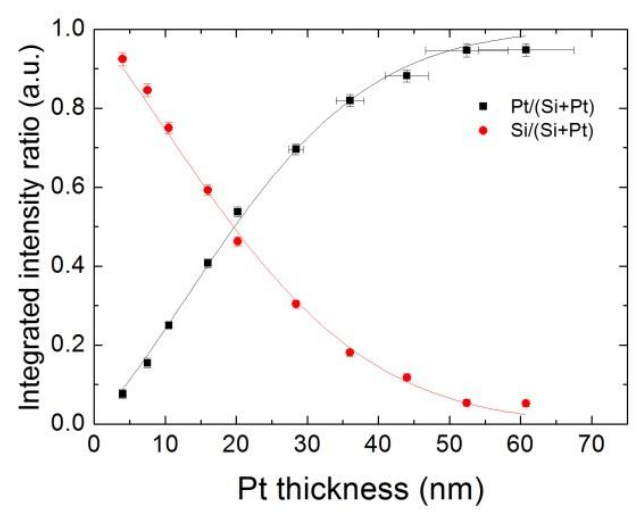

Figure 2: EDX calibration graph relating the NPI of Pt and Si to the thickness of the Pt film, for an electron beam energy of $5 \mathrm{kV}$.

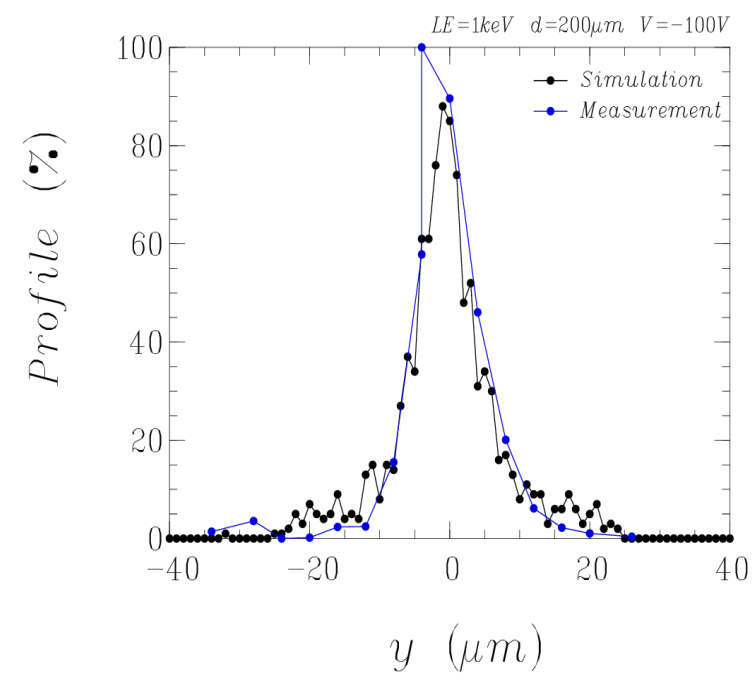

Figure 4: Example of simulated ion beam profile (black) and measured profile (blue) for a $100 \mathrm{~V}$ ion energy and $200 \mathrm{um}$ gap. Electron beam in spot mode. 